

\section{El espacio cercano y la enseñanza de las ciencias sociales en el primer ciclo de la escuela primaria}

\section{Near Space and the Teaching of Social Sciences in the First Cycle of Primary School}

\section{O espaço próximo e o ensino das Ciências Sociais no primeiro ciclo do Ensino Básico}

Nancy Palacios Mena*

Olga Lucia Romero Castro*

\begin{abstract}
Resumen
El artículo es resultado de una indagación que tiene como objetivo analizar contenidos curriculares, estrategias pedagógicas y algunos productos de aprendizaje sobre el espacio cercano en la escuela primaria. El universo empírico es el ciclo de $1^{\circ}$ a $3^{\circ}$ de dos instituciones educativas de la ciudad de Ibagué, Tolima. Se seleccionaron estos grados porque un rastreo a los lineamientos y estándares curriculares de ciencias sociales publicados por el Ministerio de Educación de Colombia permitió establecer que la noción en cuestión debería ser enseñada en este ciclo. Se pudo determinar que, aunque las características y las problemáticas del espacio cercano se constituyen como recursos de enseñanza valiosos para la escuela en general y para la enseñanza de las
\end{abstract}

ciencias sociales en particular, estas poco aparecen en los planes de área y de aula que orientan el trabajo docente. Lo anterior trae como consecuencia que los niños evidencien dificultades en la construcción de conocimientos, y el desarrollo de habilidades espaciales y temporales que involucran el espacio cercano. El trabajo de campo realizado ayudó a fundamentar los hallazgos de otras investigaciones que han planteado que la enseñanza de las ciencias sociales sigue estando desarticulada y descontextualizada de la realidad social del alumnado.

\section{Palabras clave}

didácticas de las ciencias sociales; espacio cercano; enseñanza de la geografía; escuela primaria.

* Universidad de los Andes, Bogotá, Colombia.

** Universidad del Tolima, Colombia. 


\section{Abstract}

This paper is the result of a research that aimed to analyze curricular contents, pedagogical strategies, and some learning products about near space in elementary school. The empirical universe is the 1st to 3rd cycle of two schools in Ibagué, Tolima. These grades were selected because a search of the guidelines and curricular standards of social sciences published by the Ministry of Education of Colombia revealed that the notion in question should be taught in this cycle. It was determined that, although the characteristics and problems of near space are valuable teaching resources for the school, in general, and for the teaching of social sciences, in particular, they don't appear often in the area and classroom plans that guide the teacher's work. This causes children to have difficulties in the construction of knowledge and the development of spatial and temporal skills involving near space. The fieldwork conducted helped to substantiate the findings of other researches that have suggested that the teaching of social sciences continues to be disarticulated and decontextualized from the social reality of the students is still disjointed and decontextualized from the social reality of the students.

\section{Keywords}

teaching of social sciences; near space; teaching geography; elementary school.

\section{Resumo}

O artigo é resultado de uma pesquisa que visa analisar conteúdos curriculares, estratégias pedagógicas e alguns produtos da aprendizagem sobre o espaço próximo no Ensino Básico. $O$ universo empírico é do ciclo primeiro ao terceiro de duas instituições educativas da cidade de Ibagué, Tolima. Esse ciclos foram selecionados porque um rastreio às linhas e estándares curriculares de Ciências Sociais, publicados pelo Ministério da Educação na Colômbia, permitiu estabelecer que a noção em questão deveria ser ensinada nesses ciclos. Foi possível determinar que, ainda que as características e problemas do espaço próximo estão constituídas como recursos de ensino valiosos para a escola em geral e para o ensino das Ciências Sociais, especificamente, sua presença é escassa nos planos de área e de aula que orientam o trabalho docente. Isso traz como consequência que as crianças evidenciem dificuldades na construção de conhecimentos e no desenvolvimento de habilidades espaciais e temporais que envolvem o espaço próximo. 0 trabalho de campo realizado contribuiu para fundamentar as descobertas de outras pesquisas que afirmaram que o ensino das Ciências Sociais continua a estar desarticulada e descontextualizada da realidade social dos alunos.

\section{Palavras-chave}

didáticas das Ciências Sociais; espaço próximo; ensino da geografia; Ensino Básico. 


\section{Metodología}

La investigación que da origen al artículo es de carácter cualitativo con enfoque hermenéutico, puesto que se busca la comprensión de la realidad para reflexionar sobre las dinámicas de enseñanza-aprendizaje en torno al concepto de espacio cercano. Para la recolección de la información se utilizó la técnica de análisis documental. Los documentos analizados fueron: el plan decenal de educación (MEN, 1997), la Ley General de Educación (Ley 115 de 1994), el Decreto 1002 de 1984, y los estándares y los lineamientos de ciencias sociales (MEN, 2002, 2004). También se revisaron las mallas curriculares de ciencias sociales, dos planeaciones de clase de los grados $1^{\circ}$ a $3^{\circ}$ de dos instituciones educativas de lbagué y los dibujos de niños del grado 301 de una institución educativa de Ibagué.

En los documentos de legislación educativa, el análisis se centró en la existencia y la forma de enunciación de los contenidos curriculares sobre el espacio cercano. En los documentos institucionales se tomó como referencia los planes del área de ciencias sociales, deteniéndonos en los grados $1^{\circ}$ a $3^{\circ}$ de primaria; se analizaron dos clases de dichos grados, una centrada en los contenidos espaciales y otra en los contenidos temporales. Se ocupó principalmente de analizar tanto la estructura de los planes de área y de los planes de clase, la coherencia y congruencia entre los objetivos de aprendizaje y las actividades propuestas para alcanzarlos, como su proceso evaluativo, por ello, nos detenemos en la relación entre grado, metas de aprendizaje, innovación curricular, contenidos temáticos, evaluación y recursos para el aprendizaje. Para el caso específico no se tendrá en cuenta el modelo pedagógico, que en su mayoría es constructivista, sino la propuesta de plan de clase.

Con la lectura de los dibujos se pretende reconocer e identificar la representación que hacen los niños de su espacio cercano. Para el caso específico se trabajó con 35 niños del grado 301. En una primera fase del ejercicio los estudiantes desarrollaron libremente el dibujo, utilizando los materiales necesarios. A los estudiantes se les solicitó que representaran el espacio cercano, el cual tiene relación directa con las actividades cotidianas que en este realizan sobre sus espacios inmediatos.

\section{El espacio cercano}

El concepto de espacio cercano es una forma de permitir al estudiante la comprensión compleja y epistemológica del espacio geográfico. El espacio cercano permite reconocer procesos de apropiación cotidiana de los estudiantes, y se convierte en fuente directa de aprendizaje por sus dinámicas relacional e inmediata. Es de reconocer que desde la literatura se puede identificar como elementos similares el concepto de entorno cercano y el concepto de espacio cercano:

Entorno es aquello que engloba todo lo que rodea al sujeto y su mundo más inmediato, donde el individuo se integra y convive, y en el que no es ajena la cultura. Por tanto, es más que un medio, es un ecosistema abierto a los elementos que lo componen, como organización compleja produce orden y desorden, se inscribe en una organización pluriecológica a partir de numerosas interacciones, y es el marco interactivo donde se produce la progresiva diferenciación entre el yo y el no-yo a través de las experiencias fenomenológicas. (Roca, E.R, 2003, p. 31).

El interés del presente artículo, más que generar la discusión conceptual sobre el espacio cercano, es reconocer cómo se ha abordado el concepto tanto desde la legislación, como desde los planes y el desarrollo de las clases.

La importancia del espacio cercano se debe abordar desde diferentes ámbitos:

- Como escalas de análisis del espacio geográfico: no se puede pensar en la cercanía como relación de distancia, sino más bien como interiorización y relación con el espacio. Es el espacio vivido por los estudiantes desde diferentes contextos en los que se encuentran. Así, es posible reconocer que el espacio próximo y el cercano son espacios de relación cotidiana. La secuencia de primero la casa y sucesivamente la calle, el barrio, la localidad, la comarca, el país, el Estado, el continente, el planeta y el universo obedece a la estructura con base en círculos concéntricos espaciales que tenía el área de geografía en los años setenta y ochenta. Según Roca (2003), en dicha estructura la distancia física marca las diferentes fases y está basada en el sucesivo conocimiento que tiene el alumno del espacio que lo rodea.

- Relación con la vida cotidiana: la vida cotidiana no solo es la relación que los seres humanos tienen a diario con su entorno, sino que también se ha estructurado como categoría y tendencia geográfica de comprender la relación espacio-tiempo-hombre o, en palabras de Lindón (2006, p. 370)1', "es el ámbito que da cuenta del hacer del ser humano: actividades, acciones o prácticas cotidianas".

- Constructo y articulación de saberes escolares y extraescolares: el espacio cercano permite el aprendizaje, en razón a que busca "romper las fronteras de la escuela", ya que la sociedad depende cada vez más de la cultura académica para su reproducción cultural y material. Para Mockus, Hernández, Granes, Charum y Castro (1994), los saberes escolares y extraescolares integrados surgen como opción al desconocimiento de una sociedad dinámica que se enmarca en nuevas concepciones educativas. Por lo tanto, dichos

1 Para Lindon (2006), se identifican cuatro elementos que permiten analizar la vida cotidiana: (1) desplazamientos, (2) prácticas ancladas a un lugar, (3) escenarios, (4) patrones y rutinización de las prácticas. 
saberes se estructuran mediante la interrelación práctica y entre sus actores, y las interacciones rompen la delimitación clásica del saber escolar con la concepción moderna de saber extraescolar.

Ahora bien, desde la enseñanza de las ciencias sociales, el espacio cercano permite una mayor comprensión del espacio geográfico que no implica improvisación de contenidos, ni mucho menos quitarle peso a la formación disciplinar en las ciencias sociales.

Desde el reconocimiento de las escalas de análisis del espacio, la proximidad es una de las que más permite reconocer la apropiación que logran los estudiantes, puesto que el espacio que el niño percibe, para Hannoun (1977), se basa en el egocentrismo, sincronismo e imaginación; el niño percibe su espacio pero no concibe el espacio de los adultos. De esta forma, el espacio cercano es para socializar, y permite relaciones con otros textos, otros temas y otras realidades.

Se identifica que el espacio cercano se desarrolla desde la educación primaria puesto que el niño percibe por medio de los sentidos y lo conquista con su acción. Por otra parte, hay mayor interés por la formación de actitudes, aptitudes y conceptos de manera escalar o gradual; a medida que avanza, el niño va del espacio inmediato a la construcción de una imagen del espacio local y así avanza hacia una aproximación del espacio lejano a su vida cotidiana.

Sí se realiza un análisis frente a la enseñanza del concepto de entorno cercano en el marco de la educación en Colombia, es posible identificar su profunda ausencia, en gran medida porque para las apuestas curriculares colombianas han priorizado la enseñanza de las ciencias sociales, sustentadas en temarios y no en conceptos. Así, el conocimiento del entorno cercano no aparece enunciado explícitamente como un aspecto relevante en la formación de los estudiantes, sino solamente hasta el Decreto 1002 de 1984, en el cual se establecen los planes de estudio para la educación básica primaria, secundaria y media vocacional del país.

\section{La centralidad de la acción del estudiante}

La enseñanza de las ciencias sociales las concebirá desde 1948 como integradas (geografía e historia), y las relacionará con la acción del estudiante en el proceso formativo, así, el estudiante se verá como un sujeto activo en el que se enfoca el proceso de enseñanza-aprendizaje.

La centralidad del alumno en el quehacer educativo queda expresada en la Ley 115 de 1994, o Ley General de Educación, de la siguiente forma: "Artículo 91. El alumno o educando. El alumno o educando es el centro del proceso educativo y debe participar activamente en su propia formación integral. El Proyecto Educativo Institucional reconocerá este carácter".
Esta centralidad del alumno en el proceso educativo hay que entenderla en un contexto de gran interés por superar una concepción de la enseñanza en la que lo prioritario es repetir, y pasar a otra en la que lo fundamental es que se estructuren y organicen los hechos y los datos que se presentan a los estudiantes. Todo ello ha implicado una reflexión sobre la importancia que tiene la formación en el campo de las ciencias sociales en la educación secundaria, la pertinencia y la relevancia de los ejes temáticos que se proponen, la existencia de bases conceptuales metodológicas y cognitivas para el abordaje de estos, y las posibilidades que ofrecen dichas temáticas para hacer seguimiento al trabajo de los estudiantes, de manera que se puedan diagnosticar sus avances y dificultades (Dopazo, García y Menor, 1995)

La preocupación por una enseñanza que incluya el espacio cercano es central, porque, como subraya Arias (2005), el objetivo de las ciencias sociales es la construcción gradual en el contexto de la escuela de un tipo de conocimiento válido, y pertinente, y de unos procedimientos reconocidos para acceder a la realidad social, los conocimientos y los procedimientos que podrían orientar y dar sentido a otros conocimientos: "Las ciencias sociales no solo se interesan por saber qué son las cosas y cómo son, sino que construyen un discurso orientado a la comprensión de las personas, los grupos humanos, los hechos y los fenómenos" (Benejam, 1998, p. 66).

Se identificaron cuatro aspectos relacionados entre sí, pero con algunas diferencias que es importante y útil precisar. Estos cuatro elementos se pueden comprender de manera general en el marco de la Ley 115 de 1994 y los Lineamientos curriculares de ciencias sociales (MEN, 2002). Específicamente, el artículo 13 de dicha ley determina los objetivos comunes de todos los niveles, este artículo reza "es objetivo primordial de todos y cada uno de los niveles educativos el desarrollo integral de los educandos mediante acciones estructuradas a..."(p. 4); además, la sección tercera, enfocada a la educación básica primaria en la cual se encuentra el artículo 21 , incluye 15 aspectos que generan diversos rangos de relación del niño con la sociedad.

Desde el Ministerio de Educación Nacional - MEN - se han planteado objetivos que a partir de la dinámica de la autonomía curricular se pueden potenciar en los diferentes contextos. Además, los lineamientos curriculares de ciencias sociales ${ }^{2}$ establecen ejes generadores del conocimiento, entre los que se pueden integrar los elementos mencionados. Para comprender mejor, se depuran cada uno de los cuatro elementos:

\footnotetext{
2 En el documento oficial de lineamientos, se aclara que estos no deben tomarse de ninguna manera como una verdad concluida; pretenden, más bien, suscitar e inspirar la creación en los maestros que se desempeñan en este campo del conocimiento, y, así, lograr una enseñanza más dinámica, enriquecedora y significativa para estudiantes y profesores (MEN, 2002, p. 4). Lo anterior rompe con la realidad de las escuelas colombianas en las que se tomó como referente único y exacto cada uno de los lineamientos, lo cual también fue fortalecido por la editoriales que incluían en sus textos los lineamientos al pie de la letra.
} 
1. La importancia de que los estudiantes adquieran aprendizajes que los habiliten para contribuir al bienestar de sus comunidades. La Ley 115 de 1994 establece los objetivos de la educación primaria y secundaria. Es de señalar que los referentes a la educación primaria son mucho más someros que los de la educación secundaria; así, de forma genérica, el MEN reconoce para la educación primaria: "f) La comprensión básica del medio físico, social y cultural en el nivel local, nacional y universal, de acuerdo con el desarrollo intelectual correspondiente a la edad".

Este referente deja un espectro muy amplio que no necesariamente va a incentivar en los niños el desarrollo en sus comunidades. El objetivo se estructura desde la comprensión básica del medio en diferentes escalas, pero no se incentiva a la reflexión ni a la indagación.

2. La posibilidad de que el alumnado utilice los recursos del medio en su proceso de aprendizaje, los cuales pueden constituirse en una herramienta útil para el aprendizaje.

En los documentos analizados, se reconoce en el proceso educativo la autonomía y la relevancia de los contextos escolares, permitiendo que el niño identifique las debilidades y las fortalezas de su entorno inmediato, y propenda por una transformación de este, pero la realidad de la mayoría de escuelas del país es tomar los lineamentos y estándares al pie de la letra, y no como realmente se establecieron, como un ejemplo.

3. La necesidad de construir currículos en función de las necesidades de las comunidades de los estudiantes. Se recoge la relación alumnado-entorno inmediato, lo que en la política estatal se ha llamado pertinencia, entendida como la necesidad de que los procesos de enseñanza y aprendizaje tengan coherencia con las necesidades y las problemáticas de los contextos de los estudiantes.

El currículo como propuesta cultural se construye atendiendo a la necesidad de buscar la integración de los contextos (tiempos, espacios y condiciones) en los que se generan, transmiten y transforman los conocimientos, con los contextos existenciales y vitales en los que se usa y se dota de sentido lo aprendido. Teniendo en cuenta lo anterior, se encontró lo siguiente: según la sección de áreas y modalidades del Decreto 1002 de 1984, "Artículo 9. Como elementos importantes del proceso de aprendizaje, se organizarán en los centros educativos actividades complementarias en cada área o grupo de áreas en función de los intereses y necesidades de los alumnos y de la comunidad". Según los Lineamientos curriculares de ciencias sociales, "Las características que debe tener una pregunta para ser problematizadora: pueden conectarse con los intereses y la vida cotidiana de las y los estudiantes" (MEN, 2002, p. 64). Para los Estándares curriculares de ciencias sociales,
Los estándares son un derrotero para establecer lo que nuestros niños, niñas y jóvenes deben saber y saber hacer en la escuela para comprender de manera interdisciplinaria a los seres humanos, a las sociedades, el mundo y sobre todo a su propio país y su entorno social. (MEN, 2004, p. 28).

4. La posibilidad de que la decisión de formación profesional de los alumnos esté influida por el conocimiento que tienen del medio y el convencimiento de que sus aportes pueden ayudar a la solución de las problemáticas sociales:

Artículo 10. La orientación escolar es inherente a todas las áreas y grados y debe facilitar a los alumnos la interpretación, integración y proyección de sus experiencias, en función de su desarrollo personal y social. La orientación vocacional como parte de la escolar se debe desarrollar a través de todo el proceso educativo y facilitar al estudiante el conocimiento de sus aptitudes e intereses, de las necesidades de la comunidad y de las oportunidades que le ofrece el medio, con el fin de que pueda tomar decisiones responsables sobre su futuro. (Decreto 1002 de 1984, áreas y modalidades).

La educación es un proceso continuo que permite al educando apropiarse críticamente de los saberes, competencias, actitudes y destrezas necesarios para comprender la realidad, penetrarla, valorar su universo simbólico y darle sentido a los eventos y circunstancias de su cotidianidad. No se limita al aula escolar ni a lo propuesto por un currículo. Desborda los límites de la escuela y copa todos los espacios y ambientes de la sociedad. (MEN, 1997, p. 6).

En los cuatro aspectos identificados no se enuncia la incorporación explícita del espacio cercano, pero sí es común que se señale la importancia de que los estudiantes construyan una mirada al individuo de la sociedad y su relación con el medio ambiente; asuman las formas como proceden los científicos sociales para buscar conocimientos; $y$ asuman compromisos personales y sociales a medida que avanzan en el aprendizaje, la comprensión y la apropiación de las ciencias sociales (MEN, 2004).

La oleada de transformaciones educativas que se produjeron en los años noventa incluyó algunos cambios curriculares en la enseñanza de la historia y la geografía que llevaron a revaluar la manera como se abordaban los conceptos del entorno cercano:

En la historia se intentó pasar de una historia de reyes, batallas y conquistas, a una historia del pueblo, donde aparecían colectivos hasta ese momento ignorados como las mujeres o las minorías. Igualmente, en la geografía se hicieron esfuerzos por dejar el paradigma regional que había imperado durante la mayor parte del siglo XX, y abrirse a una geografía basada en los problemas actuales, dejando atrás el memorismo y la descripción, y apostando por la reflexión, la consulta de las fuentes y los medios de comunicación. En la primaria se le 
continuó dando importancia al entorno, pero como punto de partida, haciendo numerosos "viajes" entre los distintos ámbitos espaciales, y rompiendo con el tradicional esquema de lugares concéntricos. En secundaria, fue la geografía económica y la historia discursiva las que entraron con fuerza. En este caso, se rompía la narración lineal y diacrónica de los hechos, y se pasaba a estudiar los conceptos estructurantes de la materia: causalidad, conflicto, cambio cultural, interdependencia, modificación, poder. (Roca, 2003, p. 38).

El autor resalta que el cambio suponía una transformación de los esquemas mentales que la mayoría de los docentes no estaba dispuesta a llevar a cabo, hecho que es bien capitalizado por las grandes editoriales conocedoras de este hecho, por eso transformaron sus libros en los aspectos más llamativos, como las portadas, las imágenes, los CD-ROM y la guía del profesorado (que solo siguen una minoría). Según Roca (2003), por el contrario, las pequeñas empresas apostaron por la reforma en los contenidos, y su fracaso hizo que muchas de ellas desaparecieran, otras fueran absorbidas y el resto tuvieron que cambiar de modelo. De esta forma, la mayoría de las clases continuó impartiéndose de manera conductual: "la administración optó por un incremento considerable de la burocracia, de forma que desapareció el tiempo para la preparación de esas pequeñas innovaciones voluntaristas a que tan acostumbrado estaba el profesorado de primaria” (Roca, 2003, p. 39).

Es importante reconocer que de todas las políticas educativas revisadas, los únicos que refieren de manera implícita la relación del niño con el espacio cercano son los estándares curriculares de ciencias sociales (2004). Se puede establecer que en los grados $1^{\circ}$ a $3^{\circ}$ se hace relación con el entorno cercano, pero en la medida en que se va ascendiendo en los grados van desapareciendo las relaciones (véase la tabla 1 en la que se evidencia un eje articulador y un estándar). Se observa que algunos estándares apuntan a competencias cognitivas, en segundo lugar, otros desarrollan habilidades actitudinales y, en tercer lugar, están los que apuntan a las habilidades procedimentales.

La tabla 1 incluye procesos cognitivos que se expresan principalmente en operaciones mentales, definidas por Arias (2005) como un conjunto de acciones interiorizadas, organizadas y coordinadas con las cuales se elabora la información procedente de fuentes diversas; las operaciones mentales vinculadas son visibles mediante funciones cognitivas, que son modificables por medio de acciones pedagógicas. Según Reyes (1995), los contenidos conceptuales se refieren al saber; la singularidad que presenta el tratamiento de estos en el currículo de ciencias sociales incluye hechos, conceptos, relaciones, lugares y principios. El principal objetivo de la enseñanza de estos conceptos es que el alumnado sea capaz entender el mundo en que vive, el papel del hombre en la naturaleza, la función de las instituciones sociales y la evolución de las sociedades humanas.
Para Benejam (1998), el desarrollo de operaciones mentales es más efectivo si se vincula el trabajo escolar al entorno inmediato del alumnado, puesto que el contexto cultural brinda estructuras de significado que son muy operativas, de manera que cuando el sujeto actúa, estas estructuras mentales se ponen en funcionamiento y guían los procesos de selección de la nueva información y su interpretación. Se pretende que los estudiantes vayan acercándose progresivamente al discurso propio de las ciencias sociales y que usen este discurso de manera cada vez más espontánea.

Con el desarrollo de habilidades procedimentales (tabla 2), se busca aproximar a los estudiantes al conocimiento de las ciencias sociales; así con la inclusión de lo conceptual, lo procedimental y lo actitudinal se apunta a la integralidad en la formación de los estudiantes, pues cada uno de estos compontes, y su complementariedad aporta elementos significativos tanto en la formación inicial, como en la educación primaria y secundaria.

Tabla 1. Habilidades cognitivas en los estándares curriculares de ciencias sociales de $1^{\circ}$ a $3^{\circ}$

\begin{tabular}{|l|l|}
\hline \multicolumn{1}{|c|}{ Eje articulador } & \multicolumn{1}{c|}{ Estándares } \\
\hline $\begin{array}{l}\text { Me aproximo al conocimiento } \\
\text { como científico social }\end{array}$ & $\begin{array}{l}\text { Hago preguntas sobre mí } \\
\text { y sobre las organizaciones } \\
\text { sociales a las que pertenezco } \\
\text { (familia, curso, colegio, barrio). }\end{array}$ \\
\hline $\begin{array}{l}\text { Relaciones con la historia } \\
\text { y las culturas }\end{array}$ & $\begin{array}{l}\text { Identifico y describo cambios y } \\
\text { aspectos que se mantienen en } \\
\text { mí y en las organizaciones de mi } \\
\text { entorno. }\end{array}$ \\
\hline Relaciones espaciales y & $\begin{array}{l}\text { Reconozco en mi entorno } \\
\text { cercano las huellas que } \\
\text { ambientales }\end{array}$ \\
\hline & $\begin{array}{l}\text { dejaron las comunidades } \\
\text { que locuparon en el pasado } \\
\text { (monumentos, museos, sitios de } \\
\text { conservación histórica). }\end{array}$ \\
\hline
\end{tabular}

Tabla 2. Habilidades procedimentales en los estándares curriculares de ciencias sociales de $1^{\circ}$ a $3^{\circ}$

\begin{tabular}{|l|l|}
\hline \multicolumn{1}{|c|}{ Eje articulador } & \multicolumn{1}{c|}{ Estándares } \\
\hline - Hago preguntas sobre mí \\
y sobre las organizaciones \\
sociales a las que pertenezco \\
(familia, curso, colegio, barrio).
\end{tabular}




\begin{tabular}{|l|l|}
\multicolumn{1}{|c|}{ Eje articulador } & \multicolumn{1}{c|}{ Estándares } \\
\hline & $\begin{array}{l}\text { - Me ubico en el entorno físico y } \\
\text { de representación (en mapas } \\
\text { y planos) utilizando referentes } \\
\text { espaciales como arriba, } \\
\text { abajo, dentro, fuera, derecha, } \\
\text { izquierda. }\end{array}$ \\
\hline Relaciones espacialesy & - Establezco relaciones \\
ambientales & entre los espacios físicos \\
que ocupo (salón de clase, \\
colegio, municipio, etc.) y sus \\
representaciones (mapas, \\
planos, maquetas, etc.).
\end{tabular}

Desde la perspectiva de Quinquer (1998), permitir que los estudiantes organicen y formulen las preguntas, asegura -en parte-que se involucren en su desarrollo; parte de la idea es proporcionarles experiencias adecuadas y ricas, y proponerles casos o problemas interesantes, para que, a partir de un material de trabajo, busquen por sí mismos la solución. El objetivo es el desarrollo de capacidades y destrezas ligadas a la adquisición del pensamiento formal.

La inclusión de contenidos procedimentales constituye una estrategia para hacer operativo y secuencial el aprendizaje. Los contenidos procedimentales son acciones ordenadas y dirigidas, en las cuales el aprendizaje se construye practicando (Arias, 2005). Según Arias (2005), conviene hacer uso de procedimientos en el aula y fuera de ella para indagar, estructurar y expresar problemáticas de lo social válidas para construir conocimiento. Trabajar los procedimientos implica situarnos en el ámbito del saber hacer, los cuales se refieren a la actuación y a la dimensión ejecutiva de las respuestas humanas para resolver determinadas tareas. En consecuencia, los contenidos procedimentales se organizan de lo más simple y general a lo más cercano y complejo, e implican el aprendizaje del saber hacer; se refieren al tratamiento de la información, explicación, indagación e investigación (Loste, 1995).

En lo relacionado con los contenidos actitudinales (tabla 3), su abordaje y el alcance de la mayor cantidad de logros posibles son un gran reto en la escuela colombiana, pues se ha planteado desde la Ley 115 de 1994 que la educación es formativa y un proceso integral. Lo anterior, sumado a la presencia de algunos imaginarios producto de largas décadas de violencia, hace que la formación actitudinal en los estudiantes sea una tarea necesaria y urgente. Los estándares apuntan a que los contenidos actitudinales y los valores que incorporen los escolares se ajusten a las necesidades de su población. La inclusión de los valores, las actitudes y las normas como parte integrante de los contenidos curriculares no es nueva, pero es siempre interesante, por cuanto los valores están implícita o explícitamente presentes en todo lo educativo, como advierte Pagès (1998); lo novedoso es enseñarle a los alumnos a ser conscientes de los valores que tienen, de las normas que imponen, aceptan, y de las actitudes que adoptan y estimulan en su cotidianidad.
Tabla 3. Habilidades actitudinales en los estándares curriculares de ciencias sociales de $1^{\circ}$ a $3^{\circ}$

\begin{tabular}{|l|l|}
\hline Eje articulador & \multicolumn{1}{|c|}{ Estándares } \\
\hline - Identifico situaciones cotidianas \\
que indican cumplimiento o \\
incumplimiento en las funciones \\
de algunas organizaciones \\
sociales y políticas de mi entorno. \\
\hline - Identifico factores que generan \\
cooperación y conflicto en \\
las organizaciones sociales y \\
políticas de mi entorno y explico \\
por qué lo hacen. \\
\hline - Identifico mis derechos y \\
deberes y los de otras personas \\
en las comunidades a las que \\
pertenezco.
\end{tabular}

Con la inclusión de los desempeños conceptuales, procedimentales y actitudinales, se apunta a la integralidad en la formación de los estudiantes, pues cada uno aporta elementos significativos tanto en la formación inicial, como en la educación primaria y secundaria. Así pues, los estándares curriculares propuestos por el MEN aglutinan contenidos conceptuales, procedimentales y actitudinales; con los primeros se tiene el propósito de crear condiciones de aprendizaje para que, a partir de acciones concretas de pensamiento y producción de conocimientos, los estudiantes logren la apropiación y el manejo de conceptos propios de la ciencia. Los contenidos procedimentales hacen referencia a la manera como los estudiantes se acercan a los conocimientos de las ciencias sociales; los contenidos actitudinales indican las responsabilidades que tienen como personas y miembros de la sociedad, y se asumen cuando se conocen y valoran críticamente los descubrimientos y los avances de las ciencias sociales.

\section{De la política educativa a la realidad escolar}

Como se ha venido señalando, las políticas educativas se proponen en diferentes contextos que no han sido los más aplicables a todas las realidades escolares que se desarrollan en el país; además, se ha evidenciado una falencia en la inclusión de la enseñanza y el aprendizaje del espacio cercano, el cual, cuando se incluye, solo se hace en los grados $1^{\circ}, 2^{\circ}$ y $3^{\circ}$ de primaria.

Los programas que se consultaron no demuestran un ejercicio de construcción curricular producto de la reflexión de las necesidades educativas de los estudiantes, lo que se aprecia es más una lista de temas sin 
articulación ni cohesión. Se puede evidenciar que sigue existiendo la concepción de que lo espacial y lo temporal deben ir por separado: se presentan los ejes temáticos relacionados con la categoría tiempo (historia), por un lado, y, por el otro, con la categoría espacio (geografía). Según lo planteado en los lineamientos curriculares de ciencias sociales (MEN, 2002), la primera gran dificultad que se presenta con una propuesta de ejes temáticos aislados es que se obstaculizan la articulación y la visión conjunta que se puede obtener de la problemática social a través del ingreso de otras ciencias sociales.

En la primera programación (tabla 4) no se explican los criterios que se utilizan para definir el campo científico y el histórico. En la segunda institución (tabla 5), se crearon unas mesocompetencias que aglutinaban y articulaban las distintas asignaturas por ciclos; la estrategia tenía la intencionalidad de evitar la segmentación y la repetición de temáticas, pero exigía un trabajo mancomunado de los docentes que no siempre es fácil de lograr. Esta dificultad se traduce en la incongruencia entre la mesocompetencia y los ámbitos temáticos, en los que cada área siguió trabajando de manera independiente, de tal forma que las metas propuestas con el diseño de las mesocompetencias no se han cumplido.
En el caso de esta última, si bien se introducen elementos de los documentos producidos por el Ministerio de Educación, como ciclo, ámbitos de formación y competencia (MEN, 2002), se puede apreciar que al final lo que queda es una lista de conceptos sin que haya entre ellos la más mínima conexión; pareciera que hay un interés por dar a entender que se incorporan las exigencias de la normativa educativa, desde un ejercicio meramente enunciativo y retórico.

De otro lado, Jiménez, López y Moreno (2015) subrayan la importancia de que el currículo esté centrado en problemas sociales relevantes para favorecer la formación de pensamiento reflexivo, crítico y creativo, el análisis de los valores y de las prácticas sociales, y el aprendizaje de la toma de decisiones y la resolución de problemas. Estos autores sugieren que el aula de primaria puede convertirse en un espacio para ir preparando a los niños con un énfasis en la resolución de problemas sociales del entorno, y una formación ciudadana que promueva la reflexión y la acción.

Además de lo evidenciado, se realizó la revisión de planes de clase relacionados con apropiación del espacio cercano del grado $3^{\circ}$ de primaria de dos instituciones educativas (tabla 6).

Tabla 4. Programación n. 1 espacio cercano en los grados $1^{\circ}$ a $3^{\circ}$

\section{$1^{\circ}$ \\ Percibo mi entorno. \\ Características generales del entorno \\ - Los oficios de las personas. \\ - Atención y prevención de accidentes. \\ - Cuidados con el medio ambiente.}

\section{La naturaleza}

- Lugares especiales de la localidad (parques).

- Características de los seres vivos y no vivos.

- Las plantas (silvestres-ornamentales).

- Clasificación: partes y utilidades de las plantas.

- Los animales (silvestres-domésticos).

- Impacto ambiental por las actividades humanas.

\section{$2^{\circ}$} Campo científico

Percibo mi entorno.

Órganos y sistemas del cuerpo humano

- Sistema óseo.

- Sistema digestivo.

- Sistema respiratorio.

- Sistema circulatorio.

- Sistema muscular.

La flora y la fauna

- Ciclo del agua.

- Importancia del oxígeno para los seres vivos.

La naturaleza de nuestra ciudad

- Cuidados del medio ambiente.

- Características de los seres vivos y no vivos.

- Especies de animales y su hábitat.

- Los humedales como reservas de flora y fauna.

\section{Las plantas}

- Partes de la planta.

- Funciones de las partes de la planta.

- Utilidades e importancia de las plantas.

\section{$3^{\circ}$} Campo histórico

Conozco mi ciudad.

\section{Épocas de la ciudad}

- Prehistoria de la ciudad.

- Grupos y conformación de normas.

- Aspectos sociales y culturales de la ciudad.

- Fundación de la ciudad.

\section{La localidad}

- Nombre del barrio.

- Barrios de la localidad.

- Nombre de la localidad.

- Historia de la localidad.

- Lugares de interés dentro de la localidad.

- Puntos cardinales.

\section{Me relaciono con mi ciudad}

Geografía de la ciudad.

- Planos y mapas.

- Relieve.

- Hidrografía.

- Arquitectura 
Tabla 5. Programación n. ${ }^{\circ} 2$ mesocompetencias de los grados $1^{\circ}, 2^{\circ}$ y $3^{\circ}$

\begin{tabular}{|c|c|c|c|c|}
\hline Ciclo & Mesocompetencia & $\begin{array}{c}\text { Ámbito: el espacio, el ambiente y } \\
\text { la población }\end{array}$ & $\begin{array}{c}\text { Ámbito: el poder, la economía y } \\
\text { las organizaciones sociales }\end{array}$ & Ámbito: el tiempo y las culturas \\
\hline \multirow[t]{3}{*}{ Ciclo 1} & \multirow{3}{*}{$\begin{array}{l}\text { Participar activa y } \\
\text { responsablemente en todas las } \\
\text { actividades realizadas en su } \\
\text { entorno, mostrando seguridad y } \\
\text { confianza en sí mismo, aplicando } \\
\text { normas, principios y valores } \\
\text { que permitan crear ambientes } \\
\text { propicios para una sana } \\
\text { convivencia mediante el desarrollo } \\
\text { de estrategias de enseñanza- } \\
\text { aprendizaje en el marco de la } \\
\text { pedagogía activa; proyectos } \\
\text { lúdico- pedagógicos y experiencias } \\
\text { significativas que estimulen } \\
\text { los procesos de observación, } \\
\text { exploración y experimentación } \\
\text { en su entorno inmediato, que le } \\
\text { posibiliten identificar, comparar, } \\
\text { diferenciar y clasificar los } \\
\text { conocimientos establecidos en } \\
\text { los campos de conocimiento y } \\
\text { proyectos transversales propios } \\
\text { del primer ciclo, apropiándose del } \\
\text { pensamiento lógico-matemático } \\
\text { y el proceso lecto-escritor, como } \\
\text { base para el desarrollo del PEl } \\
\text { "Educación y Trabajo". }\end{array}$} & $\begin{array}{l}\text { 10 } \\
\text { Espacio geográfico urbano y rural. } \\
\text { Características de la localidad, el } \\
\text { barrio, la escuela y la casa. Los } \\
\text { elementos naturales de fauna } \\
\text { y flora, fuentes hídricas, forma } \\
\text { de relieve. Ubicación de puntos } \\
\text { cardinales en diferentes planos. } \\
\text { los medios de transporte y señales } \\
\text { de transito. }\end{array}$ & $\begin{array}{l}\text { 10 } \\
\text { Formas de organización } \\
\text { política cercana: autoridades } \\
\text { familiares, autoridades escolares, } \\
\text { autoridades locales. Usos } \\
\text { económicos de los espacios } \\
\text { urbanos y rurales. }\end{array}$ & $\begin{array}{l}\mathbf{1 0}^{\mathbf{0}} \\
\text { Reconocimiento del ser en } \\
\text { entornos cercanos: casa, aula, } \\
\text { escuela y barrio. }\end{array}$ \\
\hline & & $\begin{array}{l}2^{\circ} \\
\text { El espacio urbano, características, } \\
\text { configuraciones, usos y } \\
\text { actividades. Habilidades } \\
\text { espaciales: observación, } \\
\text { descripción, orientación y } \\
\text { cartografía. }\end{array}$ & $\begin{array}{l}2^{\circ} \\
\text { Aproximación a formas } \\
\text { de gobierno y autoridad. } \\
\text { Comparación de formas de } \\
\text { producción económicas a través } \\
\text { del tiempo. }\end{array}$ & $\begin{array}{l}2^{\circ} \\
\text { Concepto de historia personal } \\
\text { y familiar relacionada con la } \\
\text { historia de la ciudad. Habilidades } \\
\text { temporales: fuentes históricas y } \\
\text { ubicación temporal. }\end{array}$ \\
\hline & & $\begin{array}{l}3^{\circ} \\
\text { Formas del paisaje geográfico. } \\
\text { Posición geográfica y astronómica } \\
\text { de Colombia, sus límites, clima, } \\
\text { hidrografía y formas del relieve. } \\
\text { Departamentos que conforman } \\
\text { cada región natural, sus capitales, } \\
\text { economía, hidrografía. }\end{array}$ & $\begin{array}{l}3^{\circ} \\
\text { Instancias de participación de la } \\
\text { comunidad educativa, normas de } \\
\text { socialización en su cotidianidad. } \\
\text { Organización económica y política } \\
\text { de los departamentos del país. }\end{array}$ & \\
\hline
\end{tabular}

Tabla 6. Estructuras de clases de $3^{\circ}$ de primaria

\begin{tabular}{l|l|}
\hline \multicolumn{1}{|c|}{ Institución educativa I } & \multicolumn{1}{c|}{ Institución educativa II } \\
\hline Clase: La historia y su importancia & Clase: El paisaje y sus elementos \\
Logro & Estándar \\
\hline Estándar & Competencia a desarrollar \\
Contenido & Recursos \\
\hline $\begin{array}{l}\text { Desarrollo: explicación, } \\
\text { planteamiento de ejercicios, } \\
\text { ejemplos, ejercicio en clase. }\end{array}$ & Metodología \\
\hline $\begin{array}{l}\text { Pregunta problematizadora } \\
\text { Evaluación }\end{array}$ & \\
\hline
\end{tabular}

Las clases consultadas son entregadas por los docentes a la coordinación del colegio para su respectiva revisión, sin embargo, hay que aclarar que no se evidenció que las que se consultó hubieran pasado por el proceso de revisión. Dicho examen de los planes de clase haría parte del proceso

de inspección, seguimiento y evaluación de los procesos pedagógicos internos. Las clases de ambas instituciones incluyen el eje temático, los objetivos y las activid des de aprendizaje, y la evaluación en una de ellas.

En cuanto a los objetivos de aprendizaje, en las dos instituciones aparece bajo dos enunciaciones: logro, competencias, estándar (tabla 7).

Tabla 7. Objetivos de aprendizaje

\section{Institución educativa I}

Logro: reconocer qué es la historia y su importancia en la comprensión de nuestro entorno social y cultural, e identificar las características de los periodos de la historia.

Estándar: me reconozco como ser social e histórico.

\section{Institución educativa II}

Estándar: reconozco la interacción entre el ser humano y el paisaje en diferentes contextos. Identifico las acciones económicas y las consecuencias que se derivan de esta relación.

Compet encia a desarrollar: cognitiva, actitudinal, procedimental. Reconozco conceptos generales y los relaciono con el contexto social. 
En términos de la enunciación y las formas no son las mismas; en esencia, expresan acciones que evidenciarían los aprendizajes de los estudiantes. En la primera clase, se hace una combinación de estándares y logros que desconoce que los primeros son verificables cuando se evidencia la adquisición de una competencia y el cumplimiento de un indicador de desempeño. En la segunda clase, según el planteamiento realizado, una sola enunciación apuntaría a tres competencias, que además está planteada de una manera muy general y poco aterrizada a los procesos cognitivos, biológicos y afectivos de los niños que asisten al grado $3^{\circ} \mathrm{de}$ primaria (tabla 8).

En cuanto al contenido del aprendizaje este es plano, su lectura no plantea ningún desarrollo cognitivo a los niños, hay una parte considerable de información en el contenido de la clase de historia que no podría ser comprendido por los estudiantes si no se acompañan de contenidos procedimentales en dicha dirección. Se pueden citar dos ejemplos: los contenidos de la medición del tiempo y la línea de tiempo (figura 1).

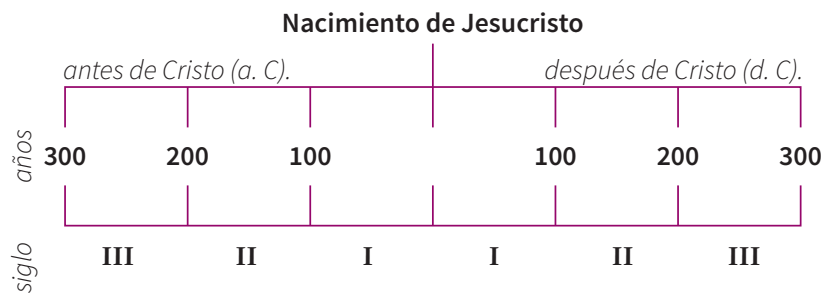

Figura 1. Ejemplo de recurso para actividad de aprendizaje

En la planeación de la clase se incluye la línea de tiempo pero no se indica una acción de construcción de conocimiento que conlleve a que los niños la comprendan. Así mismo, la clase no contiene actividades que posibiliten que lo estudiantes comprendan que un día tiene 24 horas, que una hora tiene 60 minutos. Según lo que se plantea, con que los estudiantes observen una línea de tiempo y la comenten se da por hecho que ya comprenden la medición del tiempo en antes y después de Cristo.

Lo anterior plantea un asunto central que ha sido muy cuestionado: ante la falta de una instrucción y de un entrenamiento sobre el sustento de una estrategia pedagógica adecuada, los estudiantes se limitan a repetir contenidos sin que alcancen un nivel de comprensión mínima de estos. Tal y como se presenta el contenido en las clases, las tareas de los estudiantes se reducen a operaciones mentales básicas (Saíz, 2015), saber localizar y extraer información literal de un texto académico, una fuente escrita, un mapa, un gráfico, un eje o una imagen. Solo suponen destrezas de lectura, descripción, localización, repetición, reproducción o memorización de información textual o icónica a nivel básico.

No se promueven otras actividades como localizar la idea principal, resumir la información básica ofrecida en esta actividad o hacer un esquema de ella, definir conceptos, relacionar, establecer semejanzas o diferencias entre ellos; buscar y resumir nueva información en otras fuentes; $y$, finalmente, confeccionar sencillos recursos (construir ejes cronológicos, sencillos mapas temáticos y gráficos) a partir de información básica. Tampoco se hacen actividades en las cuales se saque al estudiante de la rutina diaria del salón de clases, y se promuevan tareas de aprendizajes distintas, con las que los alumnos no solo vinculan el saber y el hacer, sino también integran la vida familiar y comunitaria a los saberes escolares.

En cuanto a las actividades de aprendizaje, siguiendo los trabajos de Palacios (2012) y Saíz (2015), las analizadas pueden ser categorizadas en un tipo uno (I), actividades que no se alejan de los métodos tradicionales de enseñanza, en las cuales los estudiantes solamente reciben información, y aquellas que implican la localización y la repetición de información. Son las que activan conocimientos declarativos de formulación literal o de base de texto o conocimientos procedimentales como meras destrezas o técnicas susceptibles de ser automatizados. Por ejemplo, se trataría de: saber localizar y extraer información literal de un texto académico, una fuente escrita, un mapa, un gráfico, un eje o una imagen. Solo suponen destrezas de lectura, descripción, localización, repetición, reproducción o memorización de información textual o icónica a nivel básico.

No se logró identificar actividades tipo dos (II), en cuyo enunciado se apunta al desarrollo de competencias, pero cuando se detallan acciones que se pide ejecutar a los estudiantes, se identifican incoherencias entre lo que se pregunta y lo que se había planteado, o las actividades a desarrollar son de un nivel procedimental o cognitivo muy básico. Aquellas que requieren comprender la información inserta en el recurso (texto académico, fuente, mapa, eje cronológico, imagen, etc.), resumiéndola, parafraseándola o esquematizándola, también inicialmente demandan contenidos procedimentales de técnicas, como los establecidos en el tipo anterior, por ejemplo, localizar la idea principal; resumir la información básica ofrecida o hacer un esquema; definir conceptos; relacionar; establecer semejanzas o diferencias entre ellos; buscar y resumir nueva información en otras fuentes; $y$, finalmente, confeccionar sencillos recursos (construir ejes cronológicos, sencillos mapas temáticos y gráficos) a partir de información básica.

Tampoco se encuentran actividades tipo tres (III), en las que hay innovaciones metodológicas y actividades de aprendizaje que apuntan al desarrollo de competencias ciudadanas. Suponen analizar, aplicar y evaluar información extraída de diferentes actividades o las que implican la creación de nueva información. Parten del nivel anteriory derivan de la resolución de preguntas inferenciales y de la aplicación de contenidos procedimentales como estrategias. Como ejemplos habría que considerar los ejercicios de empatía, simulaciones o estudios de caso; la redacción de biografías simuladas aplicando contenidos declarativos aprendidos; la valoración crítica o heurística de información proporcio- 
Tabla 8. Desarrollo de la clase

\section{Institución educativa I}

Contenido:

1. ¿Qué es el tiempo?

2. ¿Cómo podemos medir el tiempo?

3. ¿Para qué nos sirve medir el tiempo.

Desarrollo

1. Explicación del concepto el tiempo.

Tiempo: cambio y movimiento.

Ejercicio 1.

¿Te has preguntado alguna vez qué es el tiempo?

¿Cómo medían el tiempo las personas antes de que se inventaran los relojes?

¿Fecha de nacimiento de los estudiantes?

Día/mes/año/lugar.

¿Cómo podemos medir el tiempo?

¿Cómo sabemos que el tiempo pasa?

Herramientas para medir el tiempo

El tiempo es medido por: el reloj, el calendario.

¿Qué es el reloj?

Tipos de reloj. De arena, análogo, digital.

Explicar cómo funciona el reloj - am y pm

¿Para qué nos sirve organizar nuestro tiempo?

Dar ejemplos

Prever los ciclos de los vegetales, la llegada del frio

Prever la llegada de las lluvias. El crecimiento de los ríos.

Pasado presente y futuro.

Ejercicio en clase

Leer página 77 cartilla y reflexionar

Pregunta problematizara

¿Qué pasaría si las personas nunca supiera qué hora es?

Preguntas problematizadoras

¿La energía eléctrica siempre ha existido?

¿Qué pasaría si no existiera la energía eléctrica?

¿Cómo hacían nuestros antepasados para vivir?

\section{Sección}

Metodología

Se realiza una introducción a la clase con una canción de música colombiana ("Colombia es amor"). Se elabora el concepto de región desde la multiculturalidad. Se plantean las diferencias culturales, abordadas desde la música, las comidas, los trajes y las transformaciones del espacio.

Actividades

Se realiza el mapa de las regiones.

Cada estudiante, una vez ha recibido los conocimientos previos, procederá a dibujar gráficos que resalten las regiones desde la multiculturalidad.

\section{Sección}

\section{Metodología}

Se aborda un recorrido histórico, con ayuda de las fotografías y su descripción. Se les permite a los estudiantes reflexionar acerca de la fotografía y de cómo se imaginan que eran las escuelas de sus padres, abuelos y demás familiares de otras generaciones.

Actividades

Se realiza una exhibición de fotografías del paisaje y la historia de las escuelas en Colombia.

Se explican las fotografías con ayuda de las fichas técnicas.

Se socializa con los estudiantes la importancia de la escuela y su evolución histórica. 
nada por las fuentes; contrastar un mismo fenómeno en dos fuentes o diferentes interpretaciones sobre un mismo hecho; la resolución de preguntas abiertas o problemas de aprendizaje; y, finalmente, la confección de informes, autoexplicaciones e inferencias (Palacios, 2012; Saíz, 2015).

Desde la perspectiva de Sanjosé, Fernández y Vidal-Abarca (2010) y Pinzas (2007), en un trabajo sobre la jerarquización de preguntas de comprensión textual para generar conocimiento declarativo derivada de estudios sobre uso y creación de textos en el aprendizaje de ciencias, las que se le realizan a los niños en las clases que se analizaron pueden ser clasificadas como preguntas literales y de base de texto; estas se caracterizan porque tienen pistas como ¿qué?, ¿cómo? y ¿cuál?, y porque las actividades que se proponen estimulan habilidades básicas como identificar detalles, precisar espacio y tiempo de personajes, secuenciar sucesos y hechos, captar el significado de las palabras y las oraciones, y recordar pasajes y detalles de un texto.

Sobre la formación del ser, ambas clases ponen como meta de aprendizaje la reflexión, sin indicar el proceso pedagógico y los recursos didácticos que permitan que esta se suscite. Tal y como se indica en la clase, la reflexión es entendida como un proceso que brota natural y automáticamente de hacer una lectura, observar unas fotografías o ver un video. Se desconoce que la formación del ser requiere de procesos articulados de instrucción e interiorización de conceptos, habilidades y destrezas que son adquiridos cuando se promueve la construcción de conocimientos cognitivos y procedimentales.

En lo que tiene que ver con las preguntas, una de las clases propone preguntas problematizadoras, sin embargo, en una mirada detallada a estas se puede establecer que no se corresponden con la definición planteada por el MEN (2002), que define la pregunta problematizadora "como aquellas que plantean problemas con el fin, no solo de atraer la atención de las y los estudiantes sino que, su principal objetivo es fomentar la investigación constante y generar nuevos conocimientos en la clase" (p. 60). Las preguntas problematizadoras desde esta mirada son motores que impulsan la búsqueda y la creación de un nuevo saber en el aula.

En la misma línea de las actividades de aprendizaje ya analizadas, la evaluación exige a los niños solo llenar unos cuadros, ejercicio que sin duda es más producto del uso de la memoria o buscar en el libro y llenar actividades como parte del proceso de construcción de aprendizajes. El diligenciamiento del examen no solo no es producto de un proceso de mejora de aprendizajes como debe ser la evaluación, sino que no involucra operaciones de pensamiento.

A nivel general de los planes encontrados, es notorio que hay una utilización de las políticas y las propuestas educativas del MEN, pero no se evidencia autonomía curricular. Además, no hay correlación pedagógica; la forma como se estructuran los planes no se sabe a qué modelo pedagógico se refiere. Metodológicamente, no basta con decir qué acti- vidad se va a desarrollar, sino que es necesario establecer unos roles específicos, y por eso es importante saber el modelo pedagógico. Son pocas las propuestas que cuentan con objetivos claros. Los planes de clase se orientan solo a la evaluación mediante preguntas de elementos cognitivos, dejando de lado aspectos procedimentales y actitudinales. Por otro lado, no hay una definición ni interiorización del concepto de evaluación, se entiende como proceso pero se aplica como producto.

\section{Trabajo de representación del espacio cercano}

Se trabajó con 35 niños de una institución educativa de Ibaguéy se aplicó un ejercicio sobre la representación del espacio cercano, tema que los niños abordaron 8 meses atrás. El análisis de dicho ejercicio tiene como referente el trabajo de Torres y Rodríguez de Moreno (1994) ${ }^{3}$. Para el análisis de imágenes en torno al concepto de espacio cercano, se tuvieron en cuenta los siguientes elementos:

- Representación de elementos naturales y artificiales, para identificar su presencia, no por cuantificar sino por reconocer si el niño representa el espacio cercano con sus características y contextos propios.

- ¿Se incluye el ser humano? Es reconocido que el niño es egocéntrico en las representaciones que realiza del espacio; en sus entornos cercanos, él representa lo que ve y pocas veces incluye a otros seres humanos, sino que en los dibujos se representa a sí mismo.

- Actitudes que se le atribuyen al espacio y los elementos contenidos en él. Para los niños que se encuentran en los grados $1^{\circ}$ a $3^{\circ}$ es muy común identificar actitudes de agrado en el espacio, representado con caras felices a diferentes objetos como las flores, el sol y las personas.

- Relación con la cotidianidad de sus entornos cercanos. Los niños incluyen los lugares que más frecuentan como los más importantes, así es posible identificar las prácticas cotidianas ancladas a un lugar.

- Organización del espacio, ¿se ve la homogeneidad del espacio? La homogeneidad, como la representación correlacionada entre acciones y objetos, permite identificar la concepción fragmentada o no del espacio.

3 En el que se analizan dibujos realizados por niños que viven en la ciudad, en los cuales se expresa su concepto de espacio. En dicha investigación, las autoras señalan diversos elementos para analizar los dibujos (elementos del espacio geográfico cercano, lejano, diversidad de los elementos, entre otros). 
En la figura 2, se aprecia la misma estructura de representación, una casa, una letra que diferencia a cada ciudad; respecto a los elementos naturales se pueden reconocer el sol, las nubes y el suelo, no hay presencia de personas, en este caso el espacio está claramente fragmentado. La representación realizada evidencia en este estudiante que no se ha interiorizado el espacio cercano, falta desarrollar desde la escuela más habilidades geográficas, en especial la observación y la descripción.

Según Torres y Rodríguez de Moreno,

El espacio geográfico de los niños de 5 a 7 años es el vivido y experimentado. Las diferencias que establecen entre ciudad y campo son vagas y se caracterizan por criterios externos (más grande o más pequeño...), es más difícil para el niño residente en el área rural definir lo que es ciudad que al contrario. Los mapas mentales o dibujos elaborados sobre su entorno se limitan a representar una calle o parte de ella, con imágenes de casas, árboles y otros objetos que le son llamativos. (1994, p. 3).

Este aspecto se aprecia en la figura 2, en la que el estudiante separa lo urbano de lo rural, pero lo único que lo diferencia es el nombre de la ciudad.

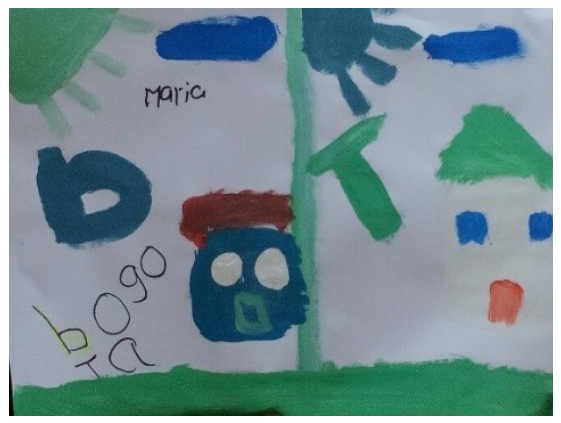

Figura 2. Representación gráfica de elementos artificiales Fuente: estudiante institución II

En cuanto a la representación no fragmentada, los estudiantes tienden a dibujar por separado diferentes elementos, pero no le imprimen orden o continuidad. Por ejemplo, en la figura 3, dibujan una persona, un árbol en el aire y un objeto que al parecer es un balón, lo que puede llevar a malas interpretaciones, puesto que se deduce que la fragmentación es resultado de la incoherencia de los dibujos.

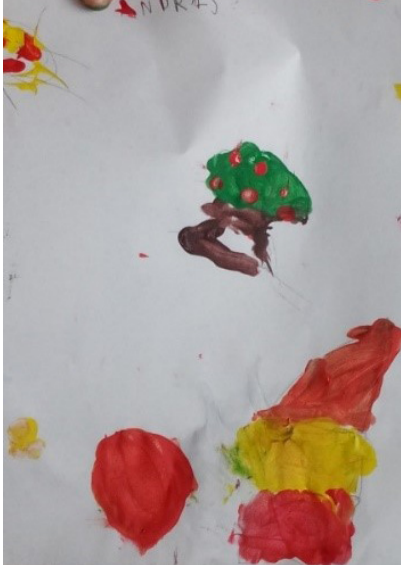

Figura 3. Representación fragmentada del espacio cercano Fuente: estudiante institución II

Los pocos estudiantes que dibujaron al ser humano, lo hicieron cantando, sonriendo, hablando, expresando aspectos de la cotidianidad de los estudiantes, elemento que indica que los estudiantes empiezan a reconocerse en su espacio.

Para Hannoun (1977), la afectividad va a tener un papel muy importante en el desarrollo del niño y su apropiación del espacio, por eso hay que brindarle afectividad para que se relacione con el medio y el hombre. Esto se ve cuando los estudiantes dibujan las personas en su entorno (figura 4) con una sonrisa en su cara, lo cual es parte de su apropiación y reconocimiento del espacio cercano. En los espacios lejanos o no apropiados se hacen representaciones artificiales y sin presencia del ser humano.

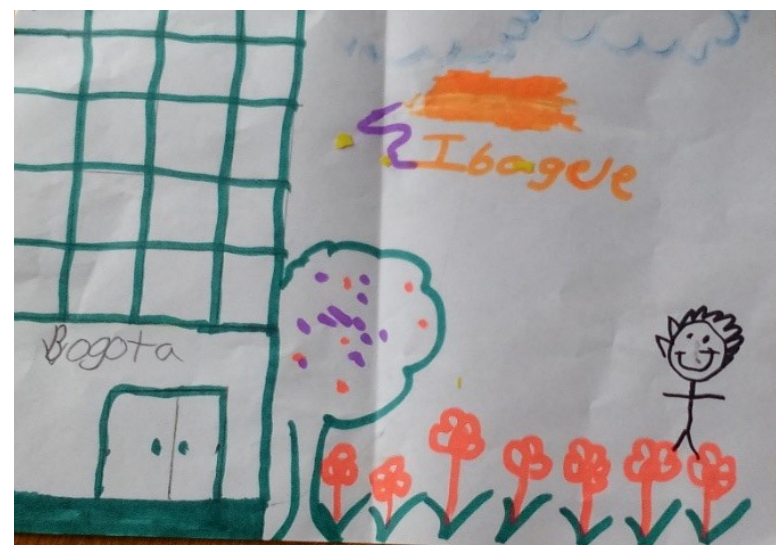

Figura 4. Actitudes del hombre en el espacio Fuente: estudiante institución I 
Otro elemento evidenciado en la falta de interiorización del concepto de espacio cercano es el hecho de que el niño represente elementos fantasiosos o imaginarios, como una jirafa o flores del tamaño de un árbol (figura 5). Si bien es cierto que no corresponden a la realidad del contexto, puede ser un intento de reconocimiento de actividades en el espacio.

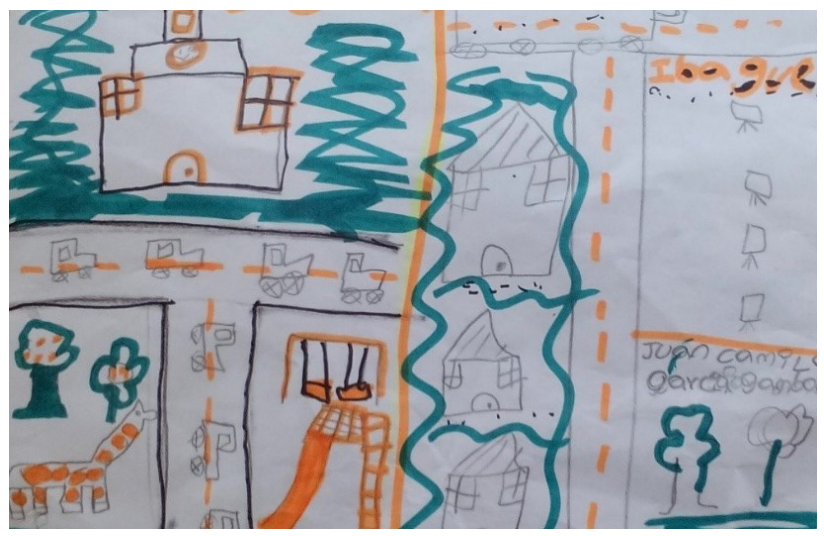

Figura 5. Representación con elementos imaginarios Fuente: estudiante institución I

De esta forma, en los dibujos realizados se evidencia que el reconocimiento del espacio cercano es complejo para los estudiantes por la falta de apropiación de este desde los saberes escolares. Los temarios escolares que se les enseñan son más del mundo de las ideas que del mundo de la realidad y cotidianidad del escolar. Es evidente la ausencia del desarrollo de habilidades geográficas, especialmente en la observación, la descripción, la representación cartográfica y el establecimiento de relaciones espaciales. En los trabajo no se logra visibilizar claramente la estructura espacial del territorio habitado por los estudiantes, concebida por Torres y Rodríguez de Moreno como "la distribución y el orden de las partes que componen todo el mapa mental, y dentro de él es posible establecer las relaciones existentes entre los elementos que los hacen dependientes unos de otros" (1994, p. 5).

Respecto a la construcción grupal de los dibujos, después de la socialización con el apoyo de un docente en formación, se realizó un dibujo colaborativo en el que se va a representar uno de sus espacios más inmediatos: el colegio, así los estudiantes representan actividades cercanas a este (flujos y fijos).

En la figura 6, se evidencia que los lugares tienen mayor detalle, se incluyen elementos que le dan connotación de una actividad específica en cada uno, se incluye el ser humano (algunos como las profesoras no corresponden a la realidad), y el espacio geográfico es más detallado y específico.

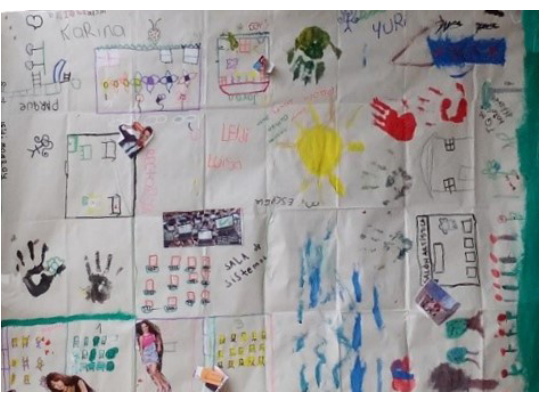

Figura 6. Representación colectiva del espacio cercano a la escuela Fuente: estudiantes institución I

De las representaciones grupales se puede reconocer que el trabajo de socialización y orientación permite mayor comprensión y reconocimiento de la acción del hombre sobre el espacio, lo que da mayor homogeneidad y relación con la realidad del contexto. El colegio se representa como un conjunto de acciones y objetos, que no solamente evidencian los usos del suelo, sino que también permiten reconocer cómo se concibe y relaciona el estudiante en un espacio cercano. La representación sigue siendo euclidiana (como en los dibujos individuales), pero también tiene intentos de representación proyectiva.

\section{Conclusiones}

El análisis permitió establecer que el concepto de espacio cercano es escasamente trabajado en el primer ciclo de la escuela primaria, aunque el currículo colombiano explícitamente contempla que se debe trabajar en estos grados. Se establece que, a pesar de que el espacio cercano se constituye como un recurso de enseñanza valioso para la escuela en general y para la enseñanza de las ciencias sociales en particular, poco aparece en los planes de área y de aula que orientan el trabajo docente. Lo anterior trae como consecuencia que los niños evidencien dificultades en la construcción de conocimientos y habilidades espaciales y temporales que involucran el espacio cercano. El trabajo de campo realizado ayudó a fundamentar los hallazgos de otros trabajos que han planteado que la enseñanza de las ciencias sociales sigue siendo desarticulada y descontextualizada de la realidad escolar del alumnado.

En la presente indagación se reconoce que hay diferencias entre las políticas educativas y la realidad escolar. El elemento a resaltar en este artículo es la invitación a reflexionar sobre la incongruencia entre las políticas educativas, el trabajo en el aula, y las necesidades educativas que las nuevas generaciones y las nuevas realidades nos demandan. $\mathrm{Si}$ bien es cierto que hace falta que en la elaboración de la política educativa se incluyan transformaciones conceptuales y epistemológicas que se abordan en las ciencias sociales hoy, también lo es la necesidad de tener estrategias pedagógicas que cuestionen las deficiencias que se hacen evidentes en dichas políticas y den un paso adelante en la construcción 
de ambientes de aprendizaje que conecten a los estudiantes con su realidad social. Conocer el contexto en el que viven es uno de los primeros pasos en la formación de ciudadanos críticos que cuestionen su cotidianidad y se convenzan de la necesidad de su aporte para transformarla.

Otro aspecto para la reflexión como resultado de la indagación realizada es el papel formativo de las universidades para los futuros docentes, porque se evidencia que en los maestros en formación hay un precario proceso de interiorización de las políticas educativas, y del manejo conceptual, pedagógico y didáctico del campo en el cual se están formando, en este caso de las ciencias sociales. En consecuencia, es necesario que en los currículos universitarios no se genere una separación del saber disciplinar del pedagógico y didáctico, por el contrario, a lo que deberíamos apuntar es a la formación de futuros profesores con una gran fortaleza disciplinar siempre en diálogo con el componente pedagógico y didáctico. Finalmente, hay que indicar que se deben incentivar procesos de investigación en el aula en los docentes, los cuales les permitan reflexionar sobre su práctica mientras se ocupan de la construcción de conocimiento y el desarrollo de habilidades de sus estudiantes, a partir de la comprensión de las problemáticas y las necesidades de su contexto.

\section{Referencias}

Arias, D. (2005). Enseñanza y aprendizaje de las ciencias sociales. Una propuesta didáctica. Bogotá: Cooperativa Editorial Magisterio.

Benejam, P. (1998). Las aportaciones de teoría sociocultural y constructivista a la enseñanza de las ciencias sociales. En P. Benejam y J. Pagès (coord.), Enseñar y aprender ciencias sociales, geografía e historia en la educación secundaria (pp. 71-95). Barcelona: Horsori/ICEUB.

Decreto 1002 de 1984 (24 de abril). Por el cual se establece el Plan de Estudios para la Educación Preescolar, Básica (primaria y secundaria) y Media Vocacional de la Educación Formal Colombiana. Diario Oficial n. 36.615.

Dopazo, M., García, J. y Menor, S. (1995). Robinsones y Colmenas. Una experiencia didáctica de geografía urbana. En IBER. Didáctica de las ciencias sociales. Diseño y unidades didácticas (pp. 33-45). Barcelona: Graó.

Hannoun, H. (1977). El niño conquista el medio. Las actividades exploradoras en la escuela primaria. Buenos aires: Editorial Kapeluz.

Jiménez, M., López, M. y Moreno, C. (2015). La formación docente en didáctica de las ciencias sociales a través de los proyectos socioeducativos en contextos de exclusión social. Revista Didáctica de las Ciencias Experimentales (29), 5, 5-23.

Ley 115 de 1994 (8 de febrero). Por la cual se expide la Ley General de Educación. Diario Oficial n. 41.214.

Lindón, A. (2006). Geografías de la vida cotidiana. En A. Lindón y D. Hiernaux, Tratado de geografía humana (pp. 357391). Barcelona: Anthropos.

Loste, M. (1995). Las unidades didácticas en el currículo de ciencias sociales de la enseñanza secundaria obligatoria. En IBER. Didáctica de las ciencias sociales. Diseño y unidades didácticas (pp. 7-15). Barcelona: Graó.

Ministerio de Educación Nacional de Colombia -MEN- (1997). Plan decenal de educación 1996-2005. Bogotá: MEN.

Ministerio de Educación Nacional de Colombia -MEN- (2002). Lineamientos curriculares de ciencias sociales. Bogotá: MEN.

Ministerio de Educación Nacional de Colombia -MEN- (2004). Estándares básicos de competencias en lenguaje, matemáticas, ciencias, y ciudadanas. Lo que los estudiantes deben saber y saber hacer con lo que aprenden. Bogotá: MEN. 
Mockus, A., Hernández, C., Granes, J., Charum, J. y Castro, M. (1994). Las fronteras de la escuela. Bogotá: Sociedad Colombiana de Pedagogía.

Pagès, J. (1998). La formación del pensamiento social. En P. Benejam Pilar y J. Pagès (coord.), Enseñar y aprender ciencias sociales, geografía e historia en la educación (pp. 151-168). Barcelona: Horsori/ICEUB.

Palacios, N. (2012). Los estándares de competencias ciudadanas en Colombia en los libros de texto. Scripta Nova: Revista Electrónica de Geografía y Ciencias Sociales, 16, 4-18.

Pinzas, J. (1997). Meta cognición y lectura. Lima: PUCP.

Quinquer, D. (1998). Estrategias de enseñanza: los métodos interactivos. En P. Benejam y J. Pagès (coord.), Enseñary aprender ciencias sociales, geografía e historia en la educación secundaria (pp. 97-121). Barcelona: Horsori/ ICEUB.

Reyes, N. (1995). Canarias en el mundo y Poeta Viana. Proyectos curriculares del área de ciencias sociales, geografía e historia. En IBER. Didáctica de las ciencias sociales. Diseño y unidades didácticas (pp. 17- 32). Barcelona: Graó.

Roca, E. (2003). Al borde del precipicio. Evolución de la educación y las ciencias sociales en el estado español, 19802002. Revista de Teoría y Didáctica de las Ciencias Sociales, 8, 31-42.

Sáiz, J. (2015). Educación histórica y narrativa nacional (tesis inédita de doctorado). Universidad de Valencia, Valencia, España.

Sanjosé, V., Fernández, J. y Vidal-Abarca, E. (2010). Importancia de las destrezas de procesa-miento de información en la comprensión de textos científicos. Revista Infancia y Aprendizaje, 33(4), 529-541.

Torres, R. y Rodríguez de Moreno, A. (1994). Dibujos infantiles y didáctica de la geografía. Bogotá. Red Academia. Universidad Pedagógica Nacional. Recuperado de file://Users/upn-21721948/Desktop/5945-15127-1-SM.pdf 